This document is the accepted manuscript version of the following article:

Perju, E., Dünki, S. J., \& Opris, D. M. (2016). A versatile synthetic path to thiol containing

polysiloxanes. Journal of Polymer Science. Part A: Polymer Chemistry, 54(18), 2940-2948.

http://doi.org/10.1002/pola.28179

\title{
A versatile synthetic path to thiol containing polysiloxanes
}

\author{
Elena Perju, ${ }^{a,+}$ Simon J. Dünki, ${ }^{\text {a,b }}$ and Dorina M. Opris ${ }^{a}$ * \\ ${ }^{a}$ Swiss Federal Laboratories for Materials Science and Technology, Laboratory of Functional Polymers, \\ Ueberlandstr. 129, CH-8600, Dübendorf, Switzerland \\ bÉcole Polytechnique Fédérale de Lausanne, Institut des Matériaux, Station 12, CH 2015, Lausanne, \\ Switzerland \\ 'Present address: "Petru Poni" Institute of Macromolecular Chemistry of Romanian Academy, Aleea Gr. \\ Ghica Voda, 41A, 700487, lasi, Romania
}

Correspondence to: Dorina M. Opris (E-mail: dorina.opris@empa.ch)

((Additional Supporting Information may be found in the online version of this article.))

\section{ABSTRACT}

A synthetic strategy to polydimethylsiloxanes and polymethylsiloxanes containing thiol functions as end- or side-groups, respectively, is presented. Such polymers are important starting materials for elastomeric networks and for post-polymerization modifications. The synthesis starts either with vinyl end-functionalized polydimethylsiloxanes or with polymethylvinylsiloxanes. Ther vinyl groups are reacted either with thioacetic acid or with a thioacetic acid/butanethiol mixture via a UV-initiated thiol-ene reaction to form the respective thioester quantitatively within few minutes. The thioesters are subsequently deprotected to the respective thiols by reduction with $\mathrm{LiAlH}_{4}$. The resulting thiol containing polysiloxanes can be used for the formation of networks or for another functionalization.

KEYWORDS: polysiloxanes, thiols, thiol-ene addition

\section{INTRODUCTION}

The reaction of thiols to double bonds with formation of thioethers, also known as thiol-ene reaction, has been widely used for the synthesis of materials with advanced properties. ${ }^{1}$ Due to its high conversion rate under mild conditions, it is not surprising that this reaction has been used in post-polymerizations as well as in polymer network formation., ${ }^{2,3}$ Additionally, it proceeds without the need of a metal catalyst, is tolerant to many functional groups, resists oxygen inhibition and has practically no side reactions. Despite of all these positive facts, the greatest disadvantage of the thiol-ene reaction is the bad odour of thiols. This problem can be overcome when thiol containing polymers (polythiols) are used since they tend to have low volatility and therefore reduced odour. ${ }^{4}$ End-functionalized polythiols are accessible via an end-group functionalization and found applications in polymer network formation. ${ }^{2}$ Side-functionalized polythiols are typically 
prepared starting from protected thiol monomers which are (co)polymerized. The formed (co)polymers are subsequently deprotected to respective polythiols, which can be further used for introduction of other functional groups. Polyamides, ${ }^{5}$ polyurethanes, ${ }^{6}$ polymethyacrylates, ${ }^{7}$ and polystyren ${ }^{8}$ containing thiols were prepared using this approach. A nice overview about synthetic strategies to polythiols is given by Nicolay et. al. ${ }^{9}$

We have recently used the thiol-ene reaction to prepare polar elastomer films in a postpolymerization starting from polymethylvinylsiloxane and mercaptopropionitrile. ${ }^{10,11}$ Apart from the bad odour of this mercapto reagent, the poor miscibility of this nonpolar polymer with the polar thiol was disadvantageous. Additionally, this postpolymerization modification required a large amount of solvent to achieve homogenous conditions. These problems might be overcome if the synthesis to the above mentioned polymer would be conducted in a reversed fashion, i.e, by starting from a polysiloxane containing thiol side groups and reacting these groups with allyl cyanide, again in a post-polymerization modification. Polysiloxanes containing thiol sidegroups are commonly prepared by condensing the hydrolysis product of mercapto dialkyloxysilane $\mathbf{A}$ with octamethylcyclotetrasiloxane $\left(\mathbf{D}_{4}\right)$ in acidic conditions $^{12}$ or by condensing $\mathbf{A}$ with dialkyloxysilane C (Scheme 1, top). However, in this way polysiloxanes of rather low molar masses form. ${ }^{13}$ Thiol end-functionalized polysiloxanes were also previously prepared. ${ }^{14}$ In a three step process starting from a hydroxyl endfunctionalized polydimethylsiloxane, the hydroxyl groups of which were converted to mesylates prior to their substitution with potassium thiolate followed by aminolysis (Scheme 1 bottom). Also in this case low molar mass polymers formed.
Despite the large variety of applications of thiol containing polysiloxanes in, for example, microfluidic devices, surface modification, as corrosion inhibitors and release agents, and in the synthesis of functional materials, the access to such polymers is unfortunately limited to low molar masses products, the synthesis of which involves multistep, time consuming reactions. ${ }^{15}$

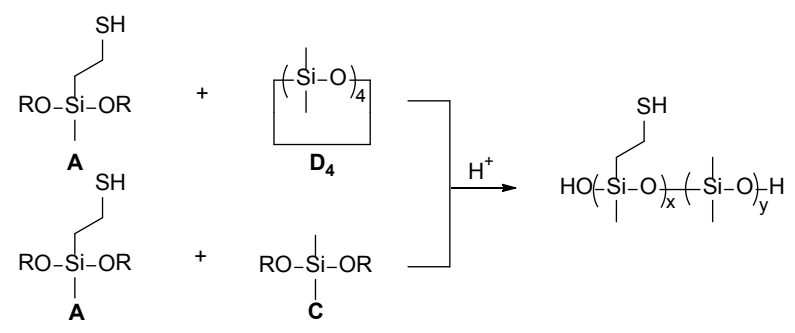

$$
\begin{aligned}
& \mathrm{HO}\left(\mathrm{H}_{2} \mathrm{C}\right)_{6} \mathrm{O}-\mathrm{Si}-\mathrm{O}-(\underset{\mid}{\mathrm{Si}}-\mathrm{O})_{\frac{\pi}{n}} \underset{l}{\mathrm{Si}}-\mathrm{O}_{(}\left(\mathrm{CH}_{2}\right)_{6} \mathrm{OH} \\
& \downarrow \mathrm{CH}_{3} \mathrm{SO}_{2} \mathrm{Cl}
\end{aligned}
$$

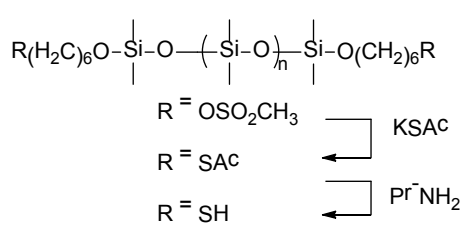

SCHEME 1 Literature approaches to polysiloxanes containing thiols as side- (top) and end-groups (bottom).

It was therefore the aim of this work to develop a synthesis pathway to polysiloxanes containing thiols as end- or side-groups which would not only be fast, simple and highly efficient but would also provide access to low as well as to high molar mass products. Particularly the latter products would suggest themselves for applications in elastomeric networks. To achieve this, we started with polysiloxanes containing vinyl groups which were used in a post-polymerization modification to introduce protected thiol groups which are subsequently used to set free the thiols on demand. The molecular weight of the resulting polymers is predetermined by the starting polymer supposed the polymer backbone is not affected by 
This document is the accepted manuscript version of the following article:

Perju, E., Dünki, S. J., \& Opris, D. M. (2016). A versatile synthetic path to thiol containing polysiloxanes. Journal of Polymer Science. Part A: Polymer Chemistry, 54(18), 2940-2948. http://doi.org/10.1002/pola.28179

the post-polymerization modifications. The UVinduced thiol-ene reaction employing thioacetic acid (1) and $\mathrm{LiAlH}_{4}$ reduction of the formed thioesters are the key reactions used.

\section{EXPERIMENTAL}

\section{Materials and methods}

Unless otherwise stated all chemicals used were reagent grade and used as received. 2,2dimethoxy-2-phenylacetophenone (DMPA), thioacetic acid (1), 1-butanethiol (2), $\mathrm{LiAlH}_{4}$, sodium hydrogen carbonate, sodium sulfate, and benzene were purchased from Sigma-Aldrich. Methanol, dichloromethane, heptane, ethyl acetate, diethyl ether $\left(\mathrm{Et}_{2} \mathrm{O}\right)$, and tetrahydrofuran (THF) were purchased from VWR. Tetramethylammonium hydroxide pentahydrate (TMAH), 1,3,5,7-tetravinyl-1,3,5,7tetramethylcyclotetrasiloxane $\left(\mathbf{V}_{\mathbf{4}}\right)$, and $\alpha, \omega$ vinyldimethylsiloxy-polydimethylsiloxane of different $M_{w}\left(\mathbf{P}_{\mathbf{t}} \mathbf{V}_{\mathrm{n}}\right.$, where $\mathbf{P}_{\mathbf{t}} \mathbf{V}_{\mathbf{1}}: M_{w}=500 \mathrm{~g} / \mathrm{mol}$, $\mathbf{P}_{\mathrm{t}} \mathbf{V}_{\mathbf{2}}: M_{w}=770 \mathrm{~g} / \mathrm{mol}, \mathbf{P}_{\mathbf{t}} \mathbf{V}_{\mathbf{3}}: M_{w}=6000 \mathrm{~g} / \mathrm{mol}$, or $P_{t} V_{4}: M_{w}=9700 \mathrm{~g} / \mathrm{mol}$ ) were purchased from ABCR. Poly(vinylmethylsiloxane) (PV) was prepared according to the literature. ${ }^{10}$ THF and $\mathrm{Et}_{2} \mathrm{O}$ were dried over sodium and distilled prior use.

${ }^{1} \mathrm{H}$ and ${ }^{13} \mathrm{C}$ NMR spectra were recorded on a Bruker Avance III 400 NMR spectrometer using a $5 \mathrm{~mm}$ BBO Prodigy ${ }^{\text {TM }}$ CryoProbe at 400.18 and 100.63 $\mathrm{MHz}$, respectively. Chemical shifts $(\delta)$ in ppm are calibrated to residual solvent peaks $\left(\mathrm{CDCl}_{3}: \delta=\right.$ 7.24 and $77.00 \mathrm{ppm})$. IR spectra were recorded on a Bruker Tensor 27 ATR FT-IR. GPC in THF were taken with an Agilent 1100 Series HPLC (Columns: serial coupled PSS SDV $5 \mathrm{u}, 100 \mathrm{~A}$ and PSS SDV $5 \mathrm{u}$, 1000A, Detector: DAD, $235 \mathrm{~nm}$ and $360 \mathrm{~nm}$; refractive index). The calibration was done with polydimethylsiloxane (PDMS) standards, while toluene was used as internal standard.

\section{1,3,5,7-tetramethyl-1,3,5,7-tetra-(2- (acetylthio)ethyl)-cyclotetrasiloxane ( $\left.V_{4} S A c\right)$}

To a solution of $\mathbf{V}_{\mathbf{4}}(20.0 \mathrm{~g}, 58 \mathrm{mmol})$ in distilled THF (250 mL), 1 (21.2 g, $280 \mathrm{mmol})$ and DMPA $(0.59 \mathrm{~g}, 2.3 \mathrm{mmol})$ were added. The reaction mixture was degassed by three freeze-pump-thaw cycles. It was then UV irradiated for $15 \mathrm{~min}$. The reaction mixture was neutralized with a saturated solution of $\mathrm{NaHCO}_{3}$, washed twice with water and extracted with dichloromethane. The combined organic layers were dried over $\mathrm{Na}_{2} \mathrm{SO}_{4}$, filtrated, and concentrated. The organic crude product was purified by flash chromatography using heptane and ethyl acetate as eluent.

${ }^{1} \mathrm{H}$ NMR $\left(400 \mathrm{MHz}, \mathrm{CDCl}_{3}, \delta\right): 0.13-0.21(\mathrm{~m}, 12 \mathrm{H}$, $\left.-\mathrm{Si}-\mathrm{CH}_{3}\right), 0.87-0.95\left(\mathrm{~m}, 7.43 \mathrm{H},-\mathrm{Si}-\mathrm{CH}_{2}-\mathrm{CH}_{2}-\mathrm{S}-\right)$, $1.23-1.28\left(\mathrm{~m}, 1.08 \mathrm{H},-\mathrm{Si}-\mathrm{CHCH}_{3}-\mathrm{S}-\right), 2.28(\mathrm{~s}$, $\left.10.83 \mathrm{H},-\mathrm{CH}_{2}-\mathrm{CH}_{2}-\mathrm{S}-\mathrm{CO}-\mathrm{C}_{3}\right), 2.31(\mathrm{~s}, 1.15 \mathrm{H},-$ $\left.\mathrm{CHCH}_{3}-\mathrm{S}-\mathrm{CO}-\mathrm{CH}_{3}\right), 2.76-2.87(\mathrm{~m}, 0.34 \mathrm{H},-\mathrm{Si}-$ $\left.\mathrm{CHCH}_{3}-\mathrm{S}-\right), 2.88-2.95\left(\mathrm{~m}, 7.34 \mathrm{H},-\mathrm{Si}-\mathrm{CH}_{2}-\mathrm{C}_{2}-\right.$ $\mathrm{S}-) ;{ }^{13} \mathrm{C}$ NMR $\left(100 \mathrm{MHz}, \mathrm{CDCl}_{3}, \delta\right):-2.40(-\mathrm{CH}-\mathrm{Si}-$ $\left.\underline{\mathrm{C}}_{3}\right),-0.64--0.56\left(-\mathrm{Si}-\underline{\mathrm{CH}}_{3}\right), 16.15\left(-\mathrm{Si}-\mathrm{CH}_{\mathrm{CH}} \mathrm{H}_{3}-\mathrm{S}-\right.$ ), $17.66-17.86\left(-\mathrm{Si}-\underline{C H}_{2}-\mathrm{CH}_{2}-\mathrm{S}-\right), 23.54$ (-Si$\left.\mathrm{CHCH}_{3}-\mathrm{S}-\right), 24.08 \quad\left(-\mathrm{Si}-\mathrm{CH}_{2}-\underline{\mathrm{CH}}_{2}-\mathrm{S}-\right), \quad 30.48 \quad(-$ $\left.\mathrm{CHCH}_{3}-\mathrm{S}-\mathrm{CO}-\underline{\mathrm{CH}}_{3}\right), 30.59\left(-\mathrm{CH}_{2}-\mathrm{CH}_{2}-\mathrm{S}-\mathrm{CO}-\underline{\mathrm{C}} \mathrm{H}_{3}\right)$, $195.78\left(-\mathrm{CHCH}_{3}-\mathrm{S}-\mathrm{CO}-\mathrm{CH}_{3}\right), 195.95\left(-\mathrm{CH}_{2}-\mathrm{CH}_{2}-\mathrm{S}-\right.$ CO- $\left.\mathrm{CH}_{3}\right)$; FTIR $\left(\mathrm{KBr}, \mathrm{v}, \mathrm{cm}^{-1}\right): 2895-2960(\mathrm{C}-\mathrm{H}$ stretch); 1658 (-C=O stretch); 1353 and 1410 ( $\delta$ C$\mathrm{H}), 1261$ and 1277 ( $\left.\delta \mathrm{Si}-\mathrm{CH}_{3}\right) ; 1058$ (Si-O-Si stretch); 790 ( $\left.\gamma \mathrm{Si}-\mathrm{CH}_{3}\right) ; 622$ (S-CO stretch); HRMS (ESI, m/z): $\left[\mathrm{M}+\mathrm{H}^{+}\right]$calcd. for $\mathrm{C}_{20} \mathrm{H}_{41} \mathrm{O}_{8} \mathrm{~S}_{4} \mathrm{Si}_{4}$ 649.0756; found 649.0746. Anal. calcd. for $\mathrm{C}_{20} \mathrm{H}_{40} \mathrm{O}_{8} \mathrm{~S}_{4} \mathrm{Si}_{4}$ : C 37.01, H 6.21; found: C 37.06, $\mathrm{H}$ 6.77. 
Synthesis of 1,3,5,7-tetramethyl-1,3,5,7-tetra-(2thioethyl)-cyclotetrasiloxane $\left(\mathrm{V}_{4} \mathrm{SH}\right)$

Reduction by $\mathrm{LiAlH}_{4}$ : To a degassed solution of $\mathrm{V}_{4} \mathrm{SAc}(2.0 \mathrm{~g}, 3.08 \mathrm{mmol})$ in dry THF $(40 \mathrm{~mL})$ a degassed solution of $\mathrm{LiAlH}_{4}(0.47 \mathrm{~g}, 12.32 \mathrm{mmol})$ in dry THF $(20 \mathrm{~mL})$ was added slowly. The reaction mixture was stirred for $1 \mathrm{~h}$ at $0{ }^{\circ} \mathrm{C}$ and for another $1 \mathrm{~h}$ at room temperature. Then a solution of acetic acid in water $(10 \%)$ was added dropwise. The organic phase was separated and washed with a saturated solution of $\mathrm{NaHCO}_{3}$, dried over $\mathrm{Na}_{2} \mathrm{SO}_{4}$, and concentrated.

Aminolysis: To a degassed solution of $\mathrm{V}_{4} \mathrm{SAc}(4.0 \mathrm{~g}$, $6.16 \mathrm{mmol})$ in dry THF $(10 \mathrm{~mL})$, butyl amine $(6 \mathrm{~mL}$, 2.5-fold molar excess with respect to thioacetate moiety) was added and stirred for $18 \mathrm{~h}$ at room temperature. Then, the solution was concentrated and chromatographed (heptane : ethyl acetate $=4$ :1) to give $\mathbf{V}_{\mathbf{4}} \mathbf{S H}$ as a colorless liquid.

${ }^{1} \mathrm{H}$ NMR $\left(400 \mathrm{MHz}, \mathrm{CDCl}_{3}, \delta\right): 0.03-0.23(\mathrm{~m}, 12 \mathrm{H}$, $\left.-\mathrm{Si}-\mathrm{CH}_{3}\right), 0.86-1.03\left(\mathrm{~m}, 8.00 \mathrm{H},-\mathrm{Si}-\mathrm{CH}_{2}-\mathrm{CH}_{2}-\mathrm{SH}\right)$, $1.23-1.42\left(\mathrm{~m}, 0.77 \mathrm{H},-\mathrm{Si}-\mathrm{CHC}_{\underline{3}}-\mathrm{SH}\right), 1.49-1.56$ (m, $4.03 \mathrm{H},-\mathrm{CH}_{2}-\mathrm{CH}_{2}-\mathrm{SH}$ and $-\mathrm{CHCH}_{3}-\mathrm{S} \underline{\mathrm{H}}$ ), $1.91-$ $2.02\left(\mathrm{~m}, 0.17 \mathrm{H},-\mathrm{Si}-\mathrm{CH}_{\mathrm{CH}}-\mathrm{SH}\right), 2.48-2.69(\mathrm{~m}$, $\left.7.73 \mathrm{H},-\mathrm{Si}-\mathrm{CH}_{2}-\mathrm{CH}_{2}-\mathrm{SH}\right) ;{ }^{13} \mathrm{C}$ NMR $(100 \mathrm{MHz}$, $\left.\mathrm{CDCl}_{3}, \delta\right):-0.39--0.33\left(-\mathrm{Si}-\mathrm{CH}_{3}\right), 19.4\left(-\mathrm{Si}-\mathrm{CH}_{2}-\right.$ $\left.\mathrm{CH}_{2}-\mathrm{SH}\right), 23.03-23.17\left(-\mathrm{Si}-\underline{\mathrm{C}} \mathrm{H}_{2}-\mathrm{CH}_{2}-\mathrm{SH}\right)$. The $\mathrm{C}$ signals for the Markovnikov product were too weak; FTIR $\left(\mathrm{KBr}, \mathrm{V}, \mathrm{cm}^{-1}\right): 2889-2958$ (C-H stretch); 2127 (S-H stretch); 1410 ( $\delta \mathrm{C}-\mathrm{H}) ; 1259$ and $1280\left(\delta \mathrm{Si}-\mathrm{CH}_{3}\right) ; 1055$ (Si-O-Si stretch); $788(\gamma$ $\left.\mathrm{Si}-\mathrm{CH}_{3}\right)$; HRMS (ESI, $\left.m / z\right)$ : $[\mathrm{M}+\mathrm{Na}]^{+}$calcd. for $\mathrm{C}_{14} \mathrm{H}_{32} \mathrm{NaO}_{4} \mathrm{~S}_{4} \mathrm{Si}_{4}, 503.0153$; found 503.0153 .

\section{Synthesis of polysiloxanes containing thioacetate} end-groups $P_{t} S A c_{n}$

$\mathbf{P}_{\mathrm{t}} \mathbf{V}_{\mathbf{n}}(10.0 \mathrm{~g})$ was dissolved in THF $(200 \mathrm{~mL})$ containing an appropriate amount of 1 (2 eq to the vinyl group in $\mathbf{P}_{\mathbf{t}} \mathbf{V}_{\mathbf{n}}$ ) and DMPA (0.01 eq). The reaction mixture was degassed and irradiated with UV light for $15 \mathrm{~min}$. Then, it was neutralized with a saturated aqueous solution of $\mathrm{NaHCO}_{3}$, washed twice with water, and extracted with dichloromethane. The organic layer was dried over $\mathrm{MgSO}_{4}$ and concentrated. The residue was purified by column chromatography on silica gel using hexane:ethyl acetate 9:1 as eluent. $\mathbf{P}_{\mathbf{t}} \mathbf{S} \mathbf{A} \mathbf{c}_{\mathbf{n}}$ were obtained as colorless viscous liquids.

$\mathrm{P}_{\mathrm{t}} \mathrm{SAc}_{1}:{ }^{1} \mathrm{H}$ NMR $\left(400 \mathrm{MHz}, \mathrm{CDCl}_{3}, \delta\right): 0.05-0.06$ $\left(\mathrm{m}, 3.68 \mathrm{H},-\mathrm{Si}-\mathrm{C}_{3}\right), 0.10-0.13\left(\mathrm{~m}, 6.07 \mathrm{H},-\mathrm{CH}_{2}-\right.$ $\mathrm{Si}-\mathrm{C}_{3}$ and $\left.-\mathrm{CH}-\mathrm{Si}-\mathrm{C}_{3}\right), 0.86-0.91(\mathrm{~m}, 1.83 \mathrm{H},-$ $\left.\mathrm{Si}-\mathrm{C}_{2}-\mathrm{CH}_{2}-\right), 1.24-1.28\left(\mathrm{~m}, 0.31 \mathrm{H},-\mathrm{Si}-\mathrm{CHC}_{3}-\right.$ $\mathrm{S}-), 2.29\left(\mathrm{~s}, 2.68 \mathrm{H},-\mathrm{CH}_{2}-\mathrm{CH}_{2}-\mathrm{S}-\mathrm{CO}-\mathrm{CH}_{3}\right), 2.32(\mathrm{~s}$, $\left.0.32 \mathrm{H},-\mathrm{CHCH}_{3}-\mathrm{S}-\mathrm{CO}-\mathrm{C}_{3}\right), 2.77-2.80(\mathrm{~m}, 0.11$ $\left.\mathrm{H},-\mathrm{Si}-\mathrm{CHCH}_{3}-\mathrm{S}-\mathrm{CO}-\right), 2.87-2.91(\mathrm{~m}, 1.80 \mathrm{H},-\mathrm{Si}-$ $\left.\mathrm{CH}_{2}-\mathrm{CH}_{2}-\mathrm{S}-\mathrm{CO}-\right) ;{ }^{13} \mathrm{C}$ NMR $\left(100 \mathrm{MHz}, \mathrm{CDCl}_{3}, \delta\right)$ : 1.25 and $-1.40\left(-\mathrm{CHCH}_{3}-\mathrm{Si}-\mathrm{CH}_{3}\right), 0.17$ and 0.31 ($\left.\mathrm{CH}_{2}-\mathrm{Si}-\underline{\mathrm{CH}}_{3}\right), 1.06-1.21\left(-\mathrm{Si}-\underline{\mathrm{CH}}_{3}\right), 16.29$ and $16.32\left(-\mathrm{Si}-\mathrm{CHCH}_{3}-\mathrm{S}-\right), 18.86$ and $18.95\left(-\mathrm{Si}-\underline{\mathrm{CH}}_{2}-\right.$ $\left.\mathrm{CH}_{2}-\mathrm{S}-\right), 24.62$ (-Si- $\left.\underline{\mathrm{C}} \mathrm{HCH}_{3}-\mathrm{S}-\right), 24.67\left(-\mathrm{Si}-\mathrm{CH}_{2}-\right.$ $\left.\mathrm{CH}_{2}-\mathrm{S}-\right), 30.55\left(-\mathrm{CHCH}_{3}-\mathrm{S}-\mathrm{CO}-\underline{C H}_{3}\right), 30.61\left(-\mathrm{CH}_{2}-\right.$ $\left.\mathrm{S}-\mathrm{CO}-\underline{\mathrm{CH}}_{3}\right), 196.26\left(-\mathrm{S}-\underline{\mathrm{CO}}-\mathrm{CH}_{3}\right) ; \mathrm{FTIR}\left(\mathrm{KBr}, \mathrm{v}, \mathrm{cm}^{-}\right.$ $\left.{ }^{1}\right)$ : 2959 ( $\mathrm{C}-\mathrm{H}$ stretch); 1688 (-C=O stretch); 1351 and $1410(\delta \mathrm{C}-\mathrm{H}), 1255\left(\delta \mathrm{Si}-\mathrm{CH}_{3}\right) ; 1031$ (Si-O-Si stretch); $792\left(\gamma \mathrm{Si}-\mathrm{CH}_{3}\right) ; 623$ (S-CO stretch).

$P_{\mathbf{t}} \mathrm{SAc}_{\mathrm{n}}:{ }^{1} \mathrm{H}$ NMR $\left(400 \mathrm{MHz}, \mathrm{CDCl}_{3}, \delta\right): 0.05-0.06$ $\left(\mathrm{m},-\mathrm{Si}-\mathrm{C}_{3}\right), 0.10-0.13\left(\mathrm{~m},-\mathrm{CH}_{2}-\mathrm{Si}-\mathrm{C}_{3}\right.$ and $\left.\mathrm{CH}-\mathrm{Si}-\mathrm{C}_{3}\right), 0.86-0.91\left(\mathrm{~m},-\mathrm{Si}-\mathrm{C}_{2}-\mathrm{CH}_{2}-\right), 1.24-$ 1.28 (m, $\left.-\mathrm{Si}-\mathrm{CHCH}_{3}-\mathrm{S}-\right)$, 2.29 (s, $-\mathrm{CH}_{2}-\mathrm{CH}_{2}-\mathrm{S}-\mathrm{CO}-$ $\left.\mathrm{C}_{3}\right), 2.32\left(\mathrm{~s},-\mathrm{CHCH}_{3}-\mathrm{S}-\mathrm{CO}-\mathrm{C}_{3}\right), 2.77-2.80(\mathrm{~m},-$ $\left.\mathrm{Si}-\mathrm{CHCH}_{3}-\mathrm{S}-\mathrm{CO}-\right)$, $2.87-2.91$ (m,-Si- $\mathrm{CH}_{2}-\mathrm{C}_{2}-\mathrm{S}-$ $\mathrm{CO}-) ;{ }^{13} \mathrm{CNMR}\left(100 \mathrm{MHz}, \mathrm{CDCl}_{3}, \delta\right):-14.8--1.24(-$ $\left.\mathrm{CHCH}_{3}-\mathrm{Si}-\underline{C H}_{3}\right), 0.15-0.31\left(-\mathrm{CH}_{2}-\mathrm{Si}-\underline{C H}_{3}\right), 0.67-$ $1.11\left(-\mathrm{Si}-\mathrm{CH}_{3}\right), 18.87-18.96\left(-\mathrm{Si}-\underline{C H}_{2}-\mathrm{CH}_{2}-\mathrm{S}-\right)$, $24.68\left(-\mathrm{Si}-\mathrm{CH}_{2}-\underline{\mathrm{CH}}_{2}-\mathrm{S}-\right)$, $30.59\left(-\mathrm{CH}_{2}-\mathrm{S}-\mathrm{CO}-\underline{\mathrm{C}} \mathrm{H}_{3}\right)$, $196.27\left(-\mathrm{S}-\mathrm{CO}-\mathrm{CH}_{3}\right)$. For the characteristics of the prepared polymers see Table 1. 
This document is the accepted manuscript version of the following article:

Perju, E., Dünki, S. J., \& Opris, D. M. (2016). A versatile synthetic path to thiol containing polysiloxanes. Journal of Polymer Science. Part A: Polymer Chemistry, 54(18), 2940-2948. http://doi.org/10.1002/pola.28179

\section{Synthesis of polysiloxanes containing thiol end- group $\left(\mathrm{P}_{t} \mathrm{SH}_{n}\right)$}

To a degassed solution of $\mathrm{P}_{\mathrm{t}} \mathrm{SAc}_{\mathrm{n}}(6.0 \mathrm{~g})$ in dry $\mathrm{Et}_{2} \mathrm{O}$ $(100 \mathrm{~mL})$ a dispersion of $\mathrm{LiAlH}_{4}(1.1$ molar ratio to the thioacetate group) in dry $\mathrm{Et}_{2} \mathrm{O}(100 \mathrm{~mL})$ was added slowly under argon atmosphere at $0{ }^{\circ} \mathrm{C}$. The reaction mixture was stirred at $0{ }^{\circ} \mathrm{C}$ for $20 \mathrm{~min}$ and at room temperature for an additional $1 \mathrm{~h}$. Then, a solution of acetic acid (10\%) was added. The phases were separated and the organic phase was washed with a saturated solution of $\mathrm{NaHCO}_{3}$. The organic layer was dried over $\mathrm{Na}_{2} \mathrm{SO}_{4}$ and concentrated to give $\mathbf{P}_{\mathrm{t}} \mathrm{SH}_{\mathrm{n}}$ as viscous liquids.

$\mathbf{P}_{\mathrm{t}} \mathrm{SH}_{1}:{ }^{1} \mathrm{H}$ NMR $\left(400 \mathrm{MHz}, \mathrm{CDCl}_{3}, \delta\right): 0.01-0.15(\mathrm{~m}$, $21.84 \mathrm{H},-\mathrm{Si}-\mathrm{C}_{3},-\mathrm{CH}_{2}-\mathrm{Si}-\mathrm{C}_{3}$ and $-\mathrm{CHCH}_{3}-\mathrm{Si}-$ $\left.\mathrm{CH}_{3}\right), 0.92-1.00\left(\mathrm{~m}, 4.00 \mathrm{H},-\mathrm{Si}-\mathrm{C}_{2}-\mathrm{CH}_{2}-\right), 1.24-$ $1.33\left(\mathrm{~m}, 0.91 \mathrm{H},-\mathrm{Si}-\mathrm{CHCH}_{3}-\mathrm{SH}\right.$ and $-\mathrm{Si}-\mathrm{CHCH}_{3}-$ $\mathrm{S} \underline{H}), 1.47-1.52\left(\mathrm{~m}, 2.05 \mathrm{H},-\mathrm{CH}_{2}-\mathrm{CH}_{2}-\mathrm{S} \underline{\mathrm{H}}\right), 1.90-$ $1.99\left(\mathrm{~m}, 0.19 \mathrm{H},-\mathrm{Si}-\mathrm{CH}_{\mathbf{H}} \mathrm{CH}_{3}-\mathrm{SH}\right), 2.55-2.63(\mathrm{~m}$, $\left.4.00 \mathrm{H},-\mathrm{Si}-\mathrm{CH}_{2}-\mathrm{CH}_{2}-\mathrm{SH}\right) ;{ }^{13} \mathrm{C} \mathrm{NMR}(100 \mathrm{MHz}$, $\left.\mathrm{CDCl}_{3}, \delta\right):-2.42--1.88\left(-\mathrm{CHCH}_{3}-\mathrm{Si}-\mathrm{CH}_{3}\right), 0.28-$ $0.42\left(-\mathrm{CH}_{2}-\mathrm{Si}-\underline{\mathrm{CH}}_{3}\right), 1.09-1.23\left(-\mathrm{Si}-\underline{\mathrm{C}} \mathrm{H}_{3}\right), 19.80(-$ $\left.\mathrm{Si}-\underline{\mathrm{C}} \mathrm{HCH}_{3}-\mathrm{SH}\right), 19.95$ (-Si- $\left.\mathrm{CH}_{2}-\underline{\mathrm{C}} \mathrm{H}_{2}-\mathrm{SH}\right), 20.12$ ($\left.\mathrm{Si}-\mathrm{CH} \mathrm{CH}_{3}-\mathrm{SH}\right), 24.56$ and $24.59\left(-\mathrm{Si}-\underline{\mathrm{CH}}_{2}-\mathrm{CH}_{2}-\mathrm{SH}\right)$; FTIR $\left(\mathrm{KBr}, \mathrm{v}, \mathrm{cm}^{-1}\right)$ : 2957 (C-H stretch); 1411 ( $\delta \mathrm{C}-$ $\mathrm{H}) ; 1254\left(\delta \mathrm{Si}-\mathrm{CH}_{3}\right) ; 1030$ (Si-O-Si stretch); $791(\nu$ $\left.\mathrm{Si}-\mathrm{CH}_{3}\right)$;

$\mathbf{P}_{\mathrm{t}} \mathrm{SH}_{\mathrm{n}}:{ }^{1} \mathrm{H}$ NMR $\left(400 \mathrm{MHz}, \mathrm{CDCl}_{3}, \delta\right): 0.06-0.18$ (m, $-\mathrm{Si}-\mathrm{C}_{3},-\mathrm{CH}_{2}-\mathrm{Si}-\mathrm{C}_{3}$ and $\left.-\mathrm{CHCH}_{3}-\mathrm{Si}-\mathrm{C}_{3}\right)$, $0.93-0.99\left(\mathrm{~m},-\mathrm{Si}-\mathrm{C}_{2}-\mathrm{CH}_{2}-\right), 1.30-1.33(\mathrm{~m},-\mathrm{Si}-$ $\left.\mathrm{CHCH}_{3}-\mathrm{SH}\right), 1.47-1.52\left(\mathrm{~m},-\mathrm{CH}_{2}-\mathrm{CH}_{2}-\mathrm{S} \underline{\mathrm{H}}\right), 1.92-$ 1.99 (m, $\left.-\mathrm{Si}-\mathrm{CH}_{\mathbf{H}} \mathrm{CH}_{3}-\mathrm{SH}\right), 2.56-2.64$ (m, $-\mathrm{Si}-\mathrm{CH}_{2}-$ $\left.\mathrm{CH}_{2}-\mathrm{SH}\right) ;{ }^{13} \mathrm{C} \mathrm{NMR}\left(100 \mathrm{MHz}, \mathrm{CDCl}_{3}, \delta\right): 0.24-0.42$ $\left(-\mathrm{CH}_{2}-\mathrm{Si}-\underline{C H}_{3}\right), 0.74-1.23\left(-\mathrm{Si}-\underline{\mathrm{C}} \mathrm{H}_{3}\right), 19.76$ (-Si$\left.\mathrm{CH}_{\underline{C}} \mathrm{H}_{3}-\mathrm{SH}\right), 19.96$ (-Si- $\left.\mathrm{CH}_{2}-\underline{C H}_{2}-\mathrm{SH}\right), 24.57$ and $24.61\left(-\mathrm{Si}-\mathrm{CH}_{2}-\mathrm{CH}_{2}-\mathrm{SH}\right)$. For the characteristics of the prepared polymers see Table 1.

\section{Polymethylsiloxanes containing thioacetate side- groups PSAc}

PV (10.0 g, $116 \mathrm{mmol})$ was dissolved in THF (200 $\mathrm{mL}$ ) containing an appropriate amount of $\mathbf{1}$ and $\mathbf{2}$ (20 mol\% excess versus the vinyl group in PV was used) and DMPA (0.01 eq). The reaction mixture was degassed and irradiated with UV light for 15 min, and then concentrated. The residue was precipitated with methanol. The precipitate was redissolved in THF and precipitated again to give PSAC $_{n}$ as viscous liquids.

PSAc $_{1}:{ }^{1} \mathrm{H}$ NMR $\left(400 \mathrm{MHz}, \mathrm{CDCl}_{3}, \delta\right): 0.07-0.16$ $\left(\mathrm{m}, 6 \mathrm{H},-\mathrm{Si}-\mathrm{C}_{3}\right), 0.85-0.92\left(\mathrm{~m}, 8.79 \mathrm{H},-\mathrm{Si}-\mathrm{C}_{2}{ }_{2}-\right.$ $\mathrm{CH}_{2}-\mathrm{S}-\mathrm{CO}-,-\mathrm{Si}-\mathrm{CH}_{2}-\mathrm{CH}_{2}-\mathrm{S}-\mathrm{CH}_{2}-$, and $-\mathrm{S}-\left(\mathrm{CH}_{2}\right)_{3}-$ $\left.\mathrm{CH}_{3}\right), 1.23-1.29\left(\mathrm{~m}, 0.42 \mathrm{H},-\mathrm{Si}-\mathrm{CHCH}_{3}-\mathrm{S}-\mathrm{CO}-\right.$ and $\left.-\mathrm{Si}-\mathrm{CHCH}_{3}-\mathrm{S}-\mathrm{CH}_{2}-\right), 1.35-1.44(\mathrm{~m}, 3.39 \mathrm{H},-$ $\left.\mathrm{CH}_{2}-\mathrm{CH}_{2}-\mathrm{CH}_{3}\right), 1.50-1.58\left(\mathrm{~m}, 3.85 \mathrm{H},-\mathrm{CH}_{2}-\mathrm{CH}_{2}-\right.$ $\left.\mathrm{CH}_{2}-\mathrm{CH}_{3}\right), 2.28\left(\mathrm{~s}, 0.73 \mathrm{H},-\mathrm{CH}_{2}-\mathrm{CH}_{2}-\mathrm{S}-\mathrm{CO}-\mathrm{C}_{3}\right)$, $2.33\left(\mathrm{~s}, 0.09 \mathrm{H},-\mathrm{CHCH}_{3}-\mathrm{S}-\mathrm{CO}-\mathrm{CH}_{3}\right), 2.48-2.55$ (m, $6.55 \mathrm{H},-\mathrm{S}-\mathrm{CH}_{2}-\left(\mathrm{CH}_{2}\right)_{2}-\mathrm{CH}_{3}$ and $-\mathrm{Si}-\mathrm{CH}_{2}-\mathrm{CH}_{2}-$ $\left.\mathrm{S}-\mathrm{CH}_{2}-\right), 2.86-2.90\left(\mathrm{~m}, 0.51 \mathrm{H},-\mathrm{Si}-\mathrm{CH}_{2}-\mathrm{CH}_{2}-\mathrm{S}-\right.$ $\mathrm{CO}-) ;{ }^{13} \mathrm{C}$ NMR $\left(100 \mathrm{MHz} \mathrm{CDCl}_{3}, \delta\right):-0.07$ (-Si$\left.\mathrm{CH}_{3}\right), 13.75\left(-\mathrm{S}-\left(\mathrm{CH}_{2}\right)_{3}-\mathrm{CH}_{3}\right), 18.36\left(-\mathrm{Si}-\underline{C H}_{2}-\mathrm{CH}_{2}-\right.$ $\mathrm{S}-\mathrm{CO}-$ and $\left.-\mathrm{Si}-\underline{\mathrm{C}} \mathrm{H}_{2}-\mathrm{CH}_{2}-\mathrm{S}-\mathrm{CH}_{2}-\right), 22.09\left(-\mathrm{CH}_{2}-\right.$ $\left.\mathrm{CH}_{2}-\mathrm{CH}_{3}\right), 24.13\left(-\mathrm{Si}-\mathrm{CH}_{2}-\mathrm{CH}_{2}-\mathrm{S}-\mathrm{CO}-\right), 26.40$ (-Si$\left.\mathrm{CH}_{2}-\mathrm{CH}_{2}-\mathrm{S}-\mathrm{CH}_{2}-\right), \quad 30.59 \quad\left(-\mathrm{CH}_{2}-\mathrm{CH}_{2}-\mathrm{S}-\mathrm{CO}-\underline{\mathrm{C}} \mathrm{H}_{3}\right)$, 31.67 and $31.73\left(-\mathrm{S}-\underline{\mathrm{CH}_{2}}-\left(\mathrm{CH}_{2}\right)_{2}-\mathrm{CH}_{3},-\mathrm{CH}_{2}-\mathrm{CH}_{2}-\right.$ $\left.\mathrm{CH}_{2}-\mathrm{CH}_{3}\right)$; FTIR $\left(\mathrm{KBr}, \mathrm{V}, \mathrm{cm}^{-1}\right): 2872-2957$ (C-H stretch); 1690 (-C=O stretch); $1353-1464$ ( $\delta \mathrm{C}-$ $\mathrm{H}), 1260$ ( $\left.\delta \mathrm{Si}-\mathrm{CH}_{3}\right) ; 999-1087$ ( $\mathrm{Si}-\mathrm{O}-\mathrm{Si}$ stretch); $766-791\left(\gamma \mathrm{Si}-\mathrm{CH}_{3}\right) ; 625$ (S-CO stretch). For the characteristics of the prepared polymers see Table 2.

\section{Polymethylsiloxanes containing thiol side-groups} $\left(\mathrm{PSH}_{n}\right)$

To a solution of $\operatorname{PSAc}_{n}(10.0 \mathrm{~g})$ in dry THF $(200 \mathrm{~mL})$ a dispersion of $\mathrm{LiAlH}_{4}$ (1.1 molar ratio) in dry THF 
$(50 \mathrm{~mL})$ was added slowly under argon atmosphere. The reaction was stirred at room temperature for $2 \mathrm{~h}$. Then, a solution of acetic acid (10\%) was slowly added to the reaction mixture. The two phases were separated, and the organic phase was washed with a saturated solution of $\mathrm{NaHCO}_{3}$. The organic layer was dried over $\mathrm{Na}_{2} \mathrm{SO}_{4}$ and concentrated to give $\mathbf{P S H}_{\mathbf{n}}$ as viscous liquids. All polymers were synthesized using the same procedure except $\mathbf{P S H}_{\mathbf{1}}$ where a mixture of $\mathrm{Et}_{2} \mathrm{O} / \mathrm{THF}$ was used as solvent.

$\mathrm{PSH}_{4}:{ }^{1} \mathrm{H}$ NMR $\left(400 \mathrm{MHz}, \mathrm{CDCl}_{3}, \delta\right): 0.08-0.22(\mathrm{~m}$, $\left.6 \mathrm{H},-\mathrm{Si}-\mathrm{C}_{3}\right), 0.86-0.92\left(\mathrm{~m}, 5.26 \mathrm{H},-\mathrm{Si}-\mathrm{CH}_{2}-\mathrm{CH}_{2}-\right.$ $\mathrm{S}-\mathrm{CH}_{2}-$, and $\left.-\mathrm{S}-\left(\mathrm{CH}_{2}\right)_{3}-\mathrm{C}_{3}\right), 0.95-0.99(\mathrm{t}, 1.72 \mathrm{H}$, $\left.-\mathrm{Si}-\mathrm{C}_{2}-\mathrm{CH}_{2}-\mathrm{SH}\right), 1.23-1.29(\mathrm{~m}, 0.27 \mathrm{H},-\mathrm{Si}-$ $\mathrm{CHCH}_{3}-\mathrm{SH}$ and $\left.-\mathrm{Si}-\mathrm{CHCH}_{3}-\mathrm{S}-\right), 1.32-1.44(\mathrm{~m}$, $\left.2.46 \mathrm{H},-\mathrm{CH}_{2}-\mathrm{CH}_{2}-\mathrm{CH}_{3}\right), 1.51-1.58(\mathrm{~m}, 3.04 \mathrm{H},-$ $\mathrm{CH}_{2}-\mathrm{C}_{2}-\mathrm{CH}_{2}-\mathrm{CH}_{3}$, and $\left.-\mathrm{CH}_{2}-\mathrm{S} \underline{\mathrm{H}}\right), 1.83-0.92$ (m, $0.12 \mathrm{H},-\mathrm{Si}-\mathrm{C}_{-} \mathrm{CH}_{3}-\mathrm{SH}$ and $\left.-\mathrm{Si}-\mathrm{CH}_{-} \mathrm{CH}_{3}-\mathrm{S}-\right), 2.48-$ $2.55\left(\mathrm{~m}, 4.28 \mathrm{H},-\mathrm{S}-\mathrm{CH}_{2}-\left(\mathrm{CH}_{2}\right)_{2}-\mathrm{CH}_{3}\right.$ and $-\mathrm{Si}-\mathrm{CH}_{2}-$ $\left.\mathrm{C}_{2}-\mathrm{S}-\mathrm{CH}_{2}-\right), 2.58-2.62\left(\mathrm{~m}, 1.56 \mathrm{H},-\mathrm{Si}-\mathrm{CH}_{2}-\mathrm{CH}_{2}-\right.$ $\mathrm{SH}) ;{ }^{13} \mathrm{C}$ NMR $\left(100 \mathrm{MHz}, \mathrm{CDCl}_{3}, \delta\right):-0.06-0.06$ ($\left.\mathrm{Si}-\underline{\mathrm{C}} \mathrm{H}_{3}\right), 13.75\left(-\mathrm{S}-\left(\mathrm{CH}_{2}\right)_{3}-\underline{\mathrm{CH}}_{3}\right), 18.34\left(-\mathrm{Si}-\underline{\mathrm{C}} \mathrm{H}_{2}-\right.$
$\left.\mathrm{CH}_{2}-\mathrm{S}-\mathrm{CH}_{2}-\right), 19.41$ (-Si- $\left.\mathrm{CH}_{2}-\underline{\mathrm{CH}}_{2}-\mathrm{SH}\right), 22.09$ ($\left.\mathrm{CH}_{2}-\mathrm{CH}_{2}-\mathrm{CH}_{3}\right), 23.69$ (-Si- $\left.\mathrm{CH}_{2}-\mathrm{CH}_{2}-\mathrm{SH}\right), 26.38$ ($\left.\mathrm{Si}-\mathrm{CH}_{2}-\underline{\mathrm{C}} \mathrm{H}_{2}-\mathrm{S}-\mathrm{CH}_{2}-\right), 31.67\left(-\mathrm{S}-\underline{\mathrm{C}} \mathrm{H}_{2}-\left(\mathrm{CH}_{2}\right)_{2}-\mathrm{CH}_{3}\right)$, $31.78\left(-\mathrm{CH}_{2}-\mathrm{CH}_{2}-\mathrm{CH}_{2}-\mathrm{CH}_{3}\right)$; FTIR $\left(\mathrm{KBr}, \mathrm{v}, \mathrm{cm}^{-1}\right)$ : 2895 - 2960 (C-H stretch); 2126 (S-H stretch); $1378-1464$ ( $\delta \mathrm{C}-\mathrm{H}) ; 1259\left(\delta \mathrm{Si}-\mathrm{CH}_{3}\right) ; 999-1084$ ( $\mathrm{Si}-\mathrm{O}-\mathrm{Si}$ stretch); 765 and $790\left(\gamma \mathrm{Si}-\mathrm{CH}_{3}\right)$. For the characteristics of the prepared polymers see Table 2. The ${ }^{1} \mathrm{H} N M R,{ }^{13} \mathrm{C} N M R$ and $2 \mathrm{D}$ spectra of all polymers can be found in SI.

\section{RESULTS AND DISCUSSION}

To achieve the goal mentioned in introduction, it was of utmost importance to prove that vinylsiloxanes can successfully be employed into UV-induced thiol-ene reactions and by this way protected thiol groups can be introduced to these silicones in a virtually quantitative manner. We thus studied the UV-induced thiol-ene addition of 1 to vinylsiloxane and the subsequent reduction of the thioester formed (Scheme 2).
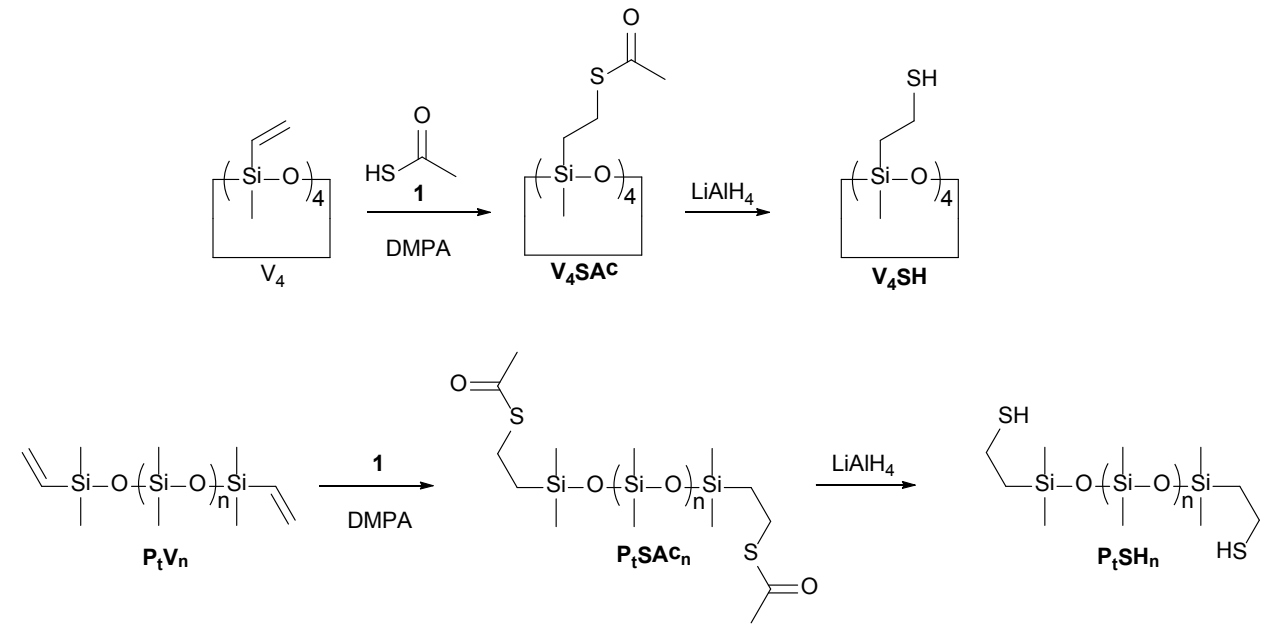

1
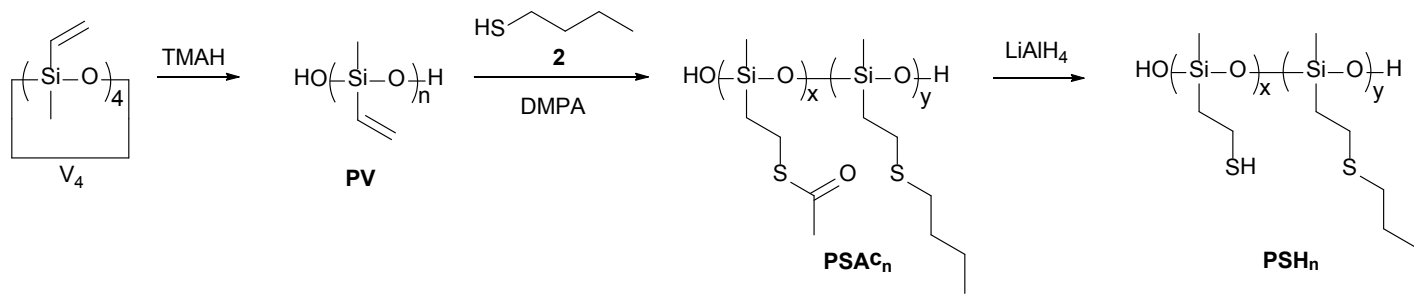
This document is the accepted manuscript version of the following article:

Perju, E., Dünki, S. J., \& Opris, D. M. (2016). A versatile synthetic path to thiol containing polysiloxanes. Journal of Polymer Science. Part A: Polymer Chemistry, 54(18), 2940-2948. http://doi.org/10.1002/pola.28179

SCHEME 2 Synthesis of a thiol containing cyclosiloxane (top), of polydimethylsiloxanes containing thiol end-groups (middle), and polymethylsiloxanes containing thiol side-groups (bottom).

For all thiol-ene reactions, the DMPA UV-initiator and a slight excess of 1 were used. The thermally induced thiol-ene reaction of $\mathbf{V}_{4}{ }^{16}$ and 1,3 divinyltetramethyldisiloxane ${ }^{17}$ with $\mathbf{1}$ and the thermally activated radical initiator azobisisobutyronitril is known. ${ }^{18}$ The main disadvantages of the reported synthesis are the long reaction time and the high temperature used. Our first orienting experiments conducted on $\mathbf{V}_{\mathbf{4}}$ showed that the vinyl groups are consumed in a relatively short time with formation of two addition products: the anti-Markovnikov (major) and the Markovnikov (minor, less than $10 \mathrm{~mol} \%$ ) products (Scheme 2). ${ }^{19,20}$ The disappearance of the vinyl protons signals between $\delta=5.75-6.15 \mathrm{ppm}$ and the appearance of a new signal $\delta=2.31 \mathrm{ppm}$ characteristic for the methyl group from thioester are clear indications for the complete reaction (See SI). The formation of $\mathbf{V}_{\mathbf{4}} \mathbf{S A c}$ was further supported by the mass spectrum which shows the $[\mathrm{M}+\mathrm{H}]^{+}$ion at $m / z=649$. Next, the deprotection of $\mathbf{V}_{4} \mathbf{S A c}$ to the thiol $\mathbf{V}_{4} \mathbf{S H}$ was conducted by reducing with $\mathrm{LiAlH}_{4}$ and found to be quantitative. The disappearance of the singlet at $\delta=2.31 \mathrm{ppm}$ characteristic for the acetyl group and the appearance of a new peak between $\delta=1.50-1.65$ ppm characteristic for the thiol groups in the ${ }^{1} \mathrm{H}$ NMR spectra are clear indications that the reduction of the thioester to thiol was successful. Furthermore, the presence of $[\mathrm{M}+\mathrm{Na}]^{+}$ion at $\mathrm{m} / \mathrm{z}=$ 503 in the MS spectrum also supports the product. Having in hand the two cyclic siloxanes $\left(\mathbf{V}_{\mathbf{4}} \mathbf{S A c}\right.$ and $\mathbf{V}_{\mathbf{4}} \mathbf{S H}$ ), their anionic and cationic polymerizations were attempted, however with only limited success. Either a low viscosity product formed, a clear indication that the polymerization was not successful, or a highly viscous polymer $\left(M_{w}=234\right.$ $\mathrm{kDa}$ ) with an unacceptable high polydispersity ( $D=$ 6.94) formed when $\mathbf{V}_{4} S A c$ was polymerized in cationic conditions.

The thiol-ene reaction of AcSH was further explored on commercially available vinyl endfunctionalized polydimethylsiloxane $\mathbf{P}_{t} \mathbf{V}_{\mathbf{n}}$ of different molar masses (Table 1) as shown in Scheme 2. Also here the conversion of vinyl groups was quantitative. Figure 1 shows the ${ }^{1} \mathrm{H}$ NMR spectra of $\mathbf{P}_{t} \mathbf{V}_{\mathbf{1}}, \mathbf{P}_{t} \mathbf{S A c _ { 1 }}$, as well as of the $\mathbf{P}_{t} \mathbf{S H}_{\mathbf{1}}$. This last polymer was obtained from the reduction of $\mathbf{P}_{\mathrm{t}} \mathrm{SAC}_{1}$ with $\mathrm{LiAlH}_{4}$. The presence of thiol protons between $1.52-1.47 \mathrm{ppm}$ is a clear indication of a successful reaction. While for the conversion of $\mathbf{P}_{t} \mathbf{S A C}$ to $\mathbf{P}_{\mathbf{t}} \mathbf{S H}$ hydrolysis in acidic or basic conditions at elevated temperatures could in principle be used, the instability of polysiloxanes under these conditions was a concern since depolymerization can occur with formation of shorter silicone chains which have uncontrolled end groups (vinyl, hydroxyl or both). To assess if there was any change in the molar mass, GPC investigations were conducted which showed a slight shift of the elugrams for $\mathbf{P}_{\mathbf{t}} \mathbf{S} \mathbf{H}_{\mathbf{n}}$ products to higher molar masses which might be due to the fact that some of the low molar mass compounds are lost during washing (Figure 2). The $M_{n}$ calculated using ${ }^{1} \mathrm{H}$ NMR spectra are in the same order of magnitude as those found by GPC. For the characteristics of the prepared polymers see Table 1.

TABLE 1 Molecular characteristics of $\mathbf{P}_{t} \mathbf{V}_{n}, \mathbf{P}_{t} \mathbf{S A c}_{n}$, and $\mathbf{P}_{\mathrm{t}} \mathbf{S H}_{\mathrm{n}}$. 


\begin{tabular}{|c|c|c|c|c|}
\hline $\begin{array}{c}\text { Sample } \\
\text { Code }\end{array}$ & $\begin{array}{c}M_{w} \\
{[\mathrm{~g} / \mathrm{mol}]}\end{array}$ & $\begin{array}{c}{ }^{2} M_{n} \\
{[\mathrm{~g} / \mathrm{mol}]}\end{array}$ & ${ }^{2} d$ & $\begin{array}{c}{ }^{3} M_{n} \text { NMR } \\
{[\mathrm{g} / \mathrm{mol}]}\end{array}$ \\
\hline$\overline{P_{t} V_{1}}$ & $500^{1}$ & - & - & 300 \\
\hline$P_{t} S_{A c}$ & - & - & - & 450 \\
\hline $\mathrm{P}_{\mathrm{t}} \mathrm{SH}_{1}$ & - & - & - & 390 \\
\hline$P_{t} V_{2}$ & $770^{1}$ & - & - & 1030 \\
\hline $\mathrm{P}_{\mathrm{t}} \mathrm{SAC}_{2}$ & - & - & - & 1140 \\
\hline $\mathrm{P}_{\mathrm{t}} \mathrm{SH}_{2}$ & - & - & - & 1190 \\
\hline $\mathbf{P}_{\mathrm{t}} \mathbf{V}_{3}$ & $\begin{array}{l}6000^{1} \\
8840^{2}\end{array}$ & 3300 & 2.7 & 5700 \\
\hline $\mathrm{P}_{\mathrm{t}} \mathrm{SAc}_{3}$ & $7000^{2}$ & 2000 & 3.47 & 6460 \\
\hline $\mathrm{P}_{\mathrm{t}} \mathrm{SH}_{3}$ & $10100^{2}$ & 5400 & 1.89 & 7840 \\
\hline$P_{t} V_{4}$ & $\begin{array}{c}9400^{1} \\
14200^{2}\end{array}$ & 8700 & 1.64 & 8580 \\
\hline $\begin{array}{l}\mathrm{P}_{\mathrm{t}} \mathrm{SAc}_{4} \\
\mathrm{P}_{\mathrm{t}} \mathrm{SH}_{4}\end{array}$ & $\begin{array}{l}12300^{2} \\
16500^{2}\end{array}$ & $\begin{array}{c}7200 \\
10900\end{array}$ & $\begin{array}{l}1.71 \\
1.51\end{array}$ & $\begin{array}{c}8650 \\
10510\end{array}$ \\
\hline
\end{tabular}

${ }^{1}$ As given by the provider; ${ }^{2}$ as determined by GPC, ${ }^{3}$ calculated from the ${ }^{1} \mathrm{H}$ NMR spectra. 


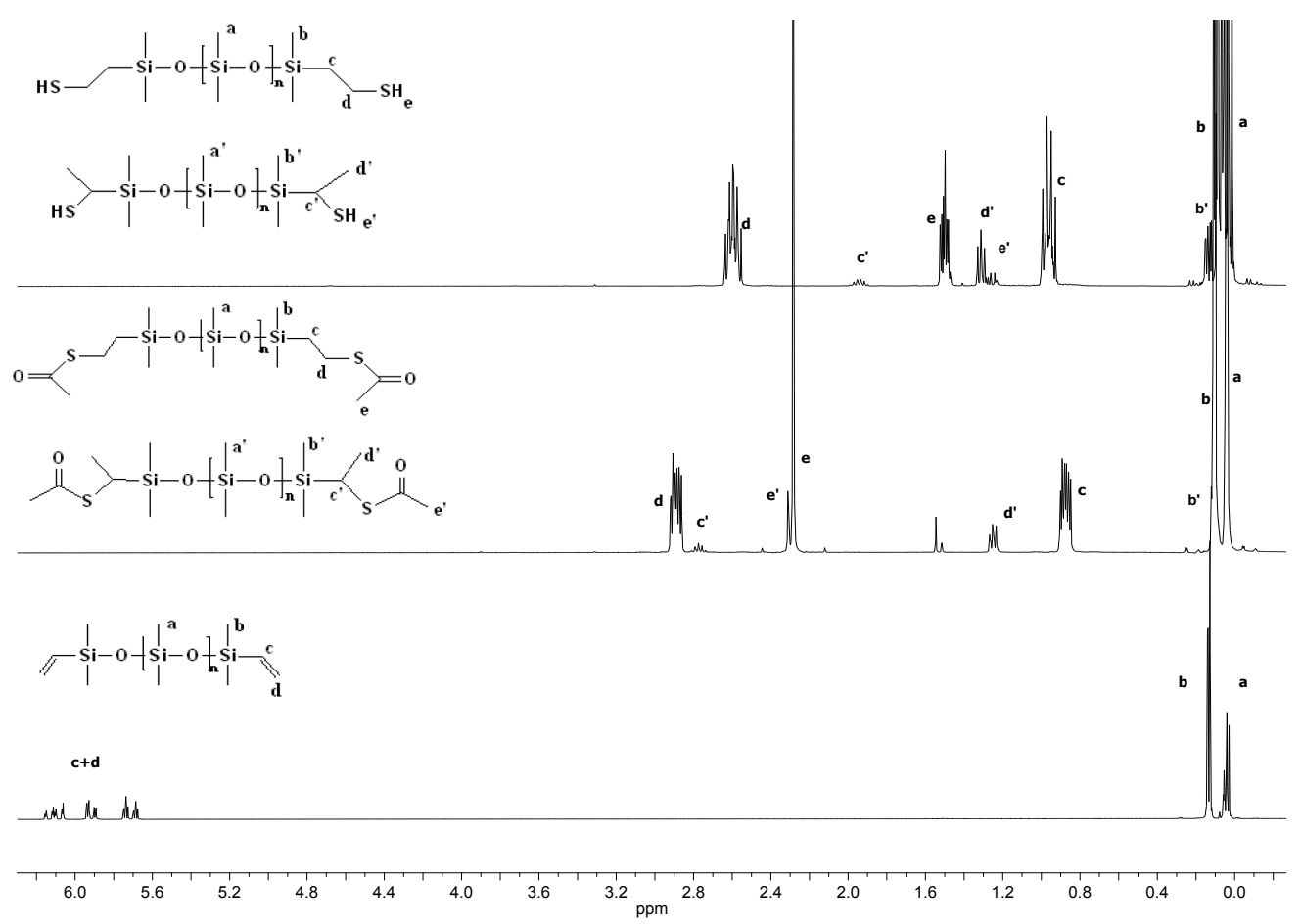

FIGURE $1{ }^{1} \mathrm{H}$ NMR spectra of $\mathbf{P}_{\mathbf{t}} \mathbf{V}_{\mathbf{1}}$ (bottom), $\mathbf{P}_{\mathbf{t}} \mathbf{S A c _ { 1 }}$ (middle), and $\mathbf{P}_{\mathrm{t}} \mathbf{S} \mathbf{H}_{1}$ (top).
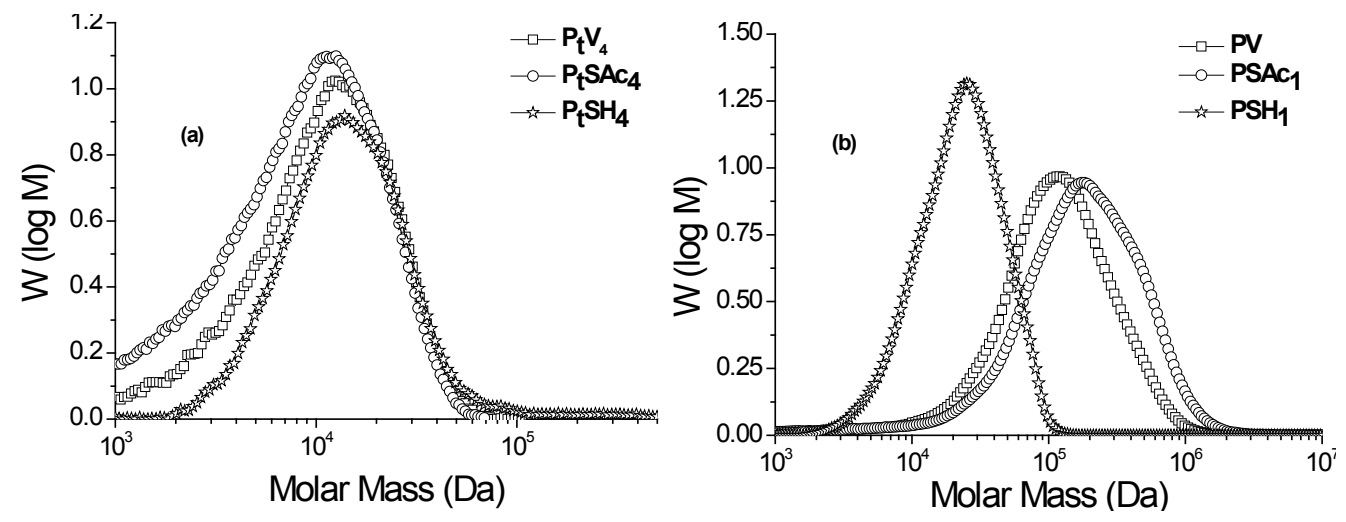

FIGURE 2 GPC elugrams of $\mathbf{P}_{t} \mathbf{V}_{4}, \mathbf{P}_{t} S_{A c}$, and $\mathbf{P}_{t} \mathbf{S H}_{4}$ (left) and of PV, $\mathbf{P S A c}$, and $\mathbf{P S H}_{1}$ (right) in THF.

For the introduction of thiols as side groups, PV $\left(M_{w}=170^{\prime} 000 \mathrm{~g} / \mathrm{mol}, M_{n}=77^{\prime} 000 \mathrm{~g} / \mathrm{mol}, D=2.2\right)$ was used. PV was prepared starting from $\mathbf{V}_{\mathbf{4}}$ via anionic polymerization in presence of $\mathrm{TMAH}^{9}{ }^{9}$ To tune the ratio of thioester side groups in $\mathbf{P S A c}_{n}, \mathbf{P V}$ which by structure carries at every repeat unit a vinyl group was reacted with different molar ratios of 1 : 2. The thioacetic acid 1 allowed us introducing protected thiol groups to the silicone chains, while 2 was used to ensure the same overall conversion of the vinyl groups and can in principle be replaced by any other functional thiol. Table 2 gives the amount of reagents used, the composition of all copolymers, as well as the molecular weights and distributions. The expected increase in the molar mass for the addition products PSAc $_{n}$ was confirmed by GPC (Figure 2). As can be seen in Figure 3, the conversion of vinyl 
groups was quantitative and new signals appeared due to the addition of $\mathbf{1}$ and $\mathbf{2}$ to the vinyl groups, however, the amount of $\mathbf{1}$ was slightly higher than expected and shows that the $\mathbf{1}$ adds faster to the vinyl groups than $\mathbf{2}$. The assignments of proton and carbon signals were possible with the help of 2D NMR spectra (Figure 4 and SI). The reduction of PSAC $_{n}$ with $\mathrm{LiAlH}_{4}$ allowed formation of polysiloxanes containing different mol\% of thiol side groups. The signal of the thiol protons overlaps with the one of the methylene protons of butyl groups as well as with the water signal and therefore, the integral of the signal between $\delta=$ $1.70-1.45$ was in all cases higher than expected. The signal intensity of the $\mathrm{C}$ atom connected to the thiol in the ${ }^{13} \mathrm{C}$ NMR spectra rises with increasing content of thiol groups in $\mathbf{P S H}_{\mathbf{n}}$ (see SI). Furthermore, the coupling of $\mathrm{SH}$ protons with the methylene groups in $\mathrm{CH}_{2}-\mathrm{SH}$ in the COSY spectrum as well as the coupling of thiol protons with the two carbons from the $\mathrm{CH}_{2}-\mathrm{CH}_{2}-\mathrm{SH}$ group in $\mathrm{HMBC}$ spectra are farther supporting the presence of thiols (see SI). GPC investigations show that the molar mass of the resulting polymers $\mathbf{P S H}_{\mathbf{n}}$ decreased. Although a decrease in molar mass after the thiol formation was expected, the molar mass was lower than that of the starting polymer
PV (Table 2). The reason behind this might be the presence of hydroxyl end-groups in $\mathbf{P S A c}_{\mathbf{n}}$ which are converted to silanolates active centres and undergo backbiting. It is known that the cyclosiloxane monomers can be polymerized in acidic and basic conditions and that the polymerization proceeds until an equilibrium is reached between polymers and cycles. ${ }^{21}$ The position of the equilibrium is however shifted to cycles when solvents and elevated temperatures are used. Despite using low temperatures for the deprotection, it was not possible to completely suppress the depolymerization which is favoured by the presence of silanolates end groups and tetrahydrofuran solvent. Nevertheless, hydroxyl end-groups functionalized polysiloxanes $\mathbf{P S H}_{\mathbf{n}}$ of appreciable molar mass and containing varying amounts of thiol side groups were prepared. Interestingly, the polydispersity of the polymers was rather low $(D<1.8)$ (Table 2$)$. One concern was the stability of the deprotected thiols which can rather easily form disulfides which leads to cross-linking. Fortunately, $\mathbf{P S H}_{\mathbf{n}}$ were stable for several months when stored in the deep fridge in dark and no change in the NMR spectra was detected.

TABLE 2 The amounts of reagents used for the synthesis of PSAC $\mathbf{n}$ and their composition, molecular weights and distributions.

\begin{tabular}{|c|c|c|c|c|c|c|c|c|c|c|c|}
\hline \multirow[t]{2}{*}{ Sample } & \multicolumn{2}{|c|}{ PV } & \multicolumn{2}{|c|}{1} & \multicolumn{2}{|r|}{2} & \multirow[t]{2}{*}{$1: 2^{\text {a) }}$} & \multirow[t]{2}{*}{$1: 2^{D \prime}$} & \multirow{2}{*}{$\begin{array}{c}M_{w}{ }^{\mathrm{C}} \\
{[\mathrm{g} / \mathrm{mol}]}\end{array}$} & \multirow{2}{*}{$\begin{array}{c}M_{n}^{\mathrm{c})} \\
{[\mathrm{g} / \mathrm{mol}]}\end{array}$} & \multirow[t]{2}{*}{$\left.P D I^{c}\right)$} \\
\hline & [g] & [mmol] & [g] & [mmol] & [g] & {$[\mathrm{mmol}]$} & & & & & \\
\hline PSAC $_{1}$ & 10 & 116 & 1.06 & 13.9 & 11.3 & 125.0 & $1: 9$ & $1: 6.4$ & 270000 & 85000 & 3.18 \\
\hline $\mathrm{PSAC}_{2}$ & 10 & 116 & 2.12 & 27.8 & 10.0 & 111.4 & $2: 8$ & $2: 6.3$ & 281000 & 89200 & 3.15 \\
\hline $\mathrm{PSAc}_{3}$ & 10 & 116 & 3.18 & 41.8 & 8.79 & 97.5 & $3: 7$ & $3: 6.1$ & 220400 & 65200 & 3.38 \\
\hline $\mathrm{PSAc}_{4}$ & 10 & 116 & 4.24 & 55.7 & 7.53 & 83.5 & $4: 6$ & $4: 5.2$ & 295100 & 105800 & 2.79 \\
\hline $\mathrm{PSAc}_{5}$ & 5 & 58 & 2.65 & 34.8 & 3.14 & 34.8 & $1: 1$ & $1: 0.64$ & 279400 & 89400 & 3.13 \\
\hline $\mathrm{PSH}_{1}$ & 10 & 5.73 & - & - & - & - & - & & 26800 & 14900 & 1.80 \\
\hline $\mathrm{PSH}_{2}$ & 10 & 11.55 & - & - & - & - & - & & 7500 & 4400 & 1.70 \\
\hline $\mathrm{PSH}_{3}$ & 10 & 17.48 & - & - & - & - & - & & 9900 & 5700 & 1.72 \\
\hline $\mathrm{PSH}_{4}$ & 10 & 23.47 & - & - & - & - & - & & 11900 & 7300 & 1.63 \\
\hline $\mathrm{PSH}_{5}$ & 6 & 17.75 & - & - & - & - & - & & 11400 & 6800 & 1.68 \\
\hline
\end{tabular}




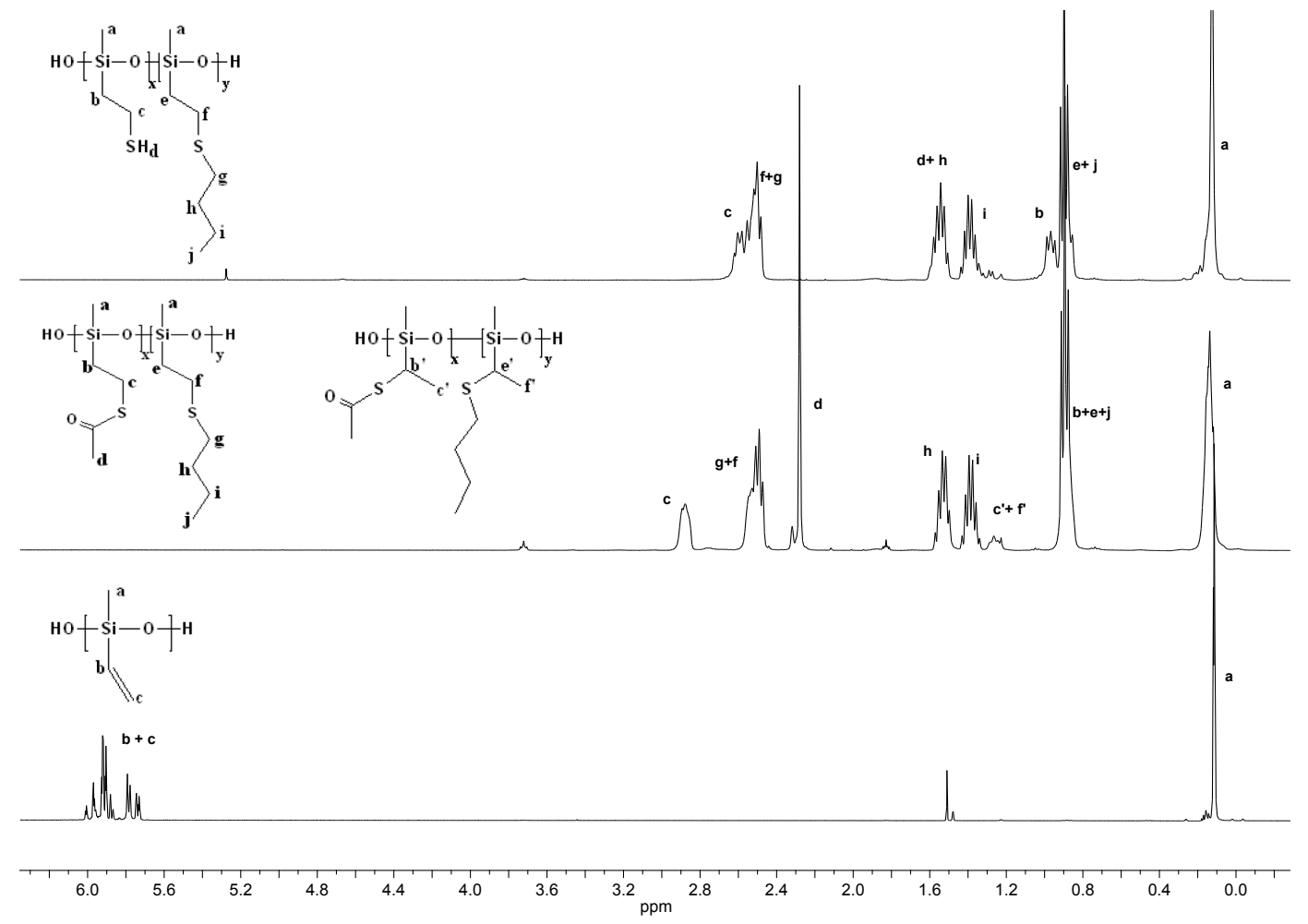

FIGURE $3{ }^{1} \mathrm{H}$ NMR spectra of PV (bottom), $\mathrm{PSAc}_{4}$ (middle), and $\mathrm{PSH}_{4}$ (top).

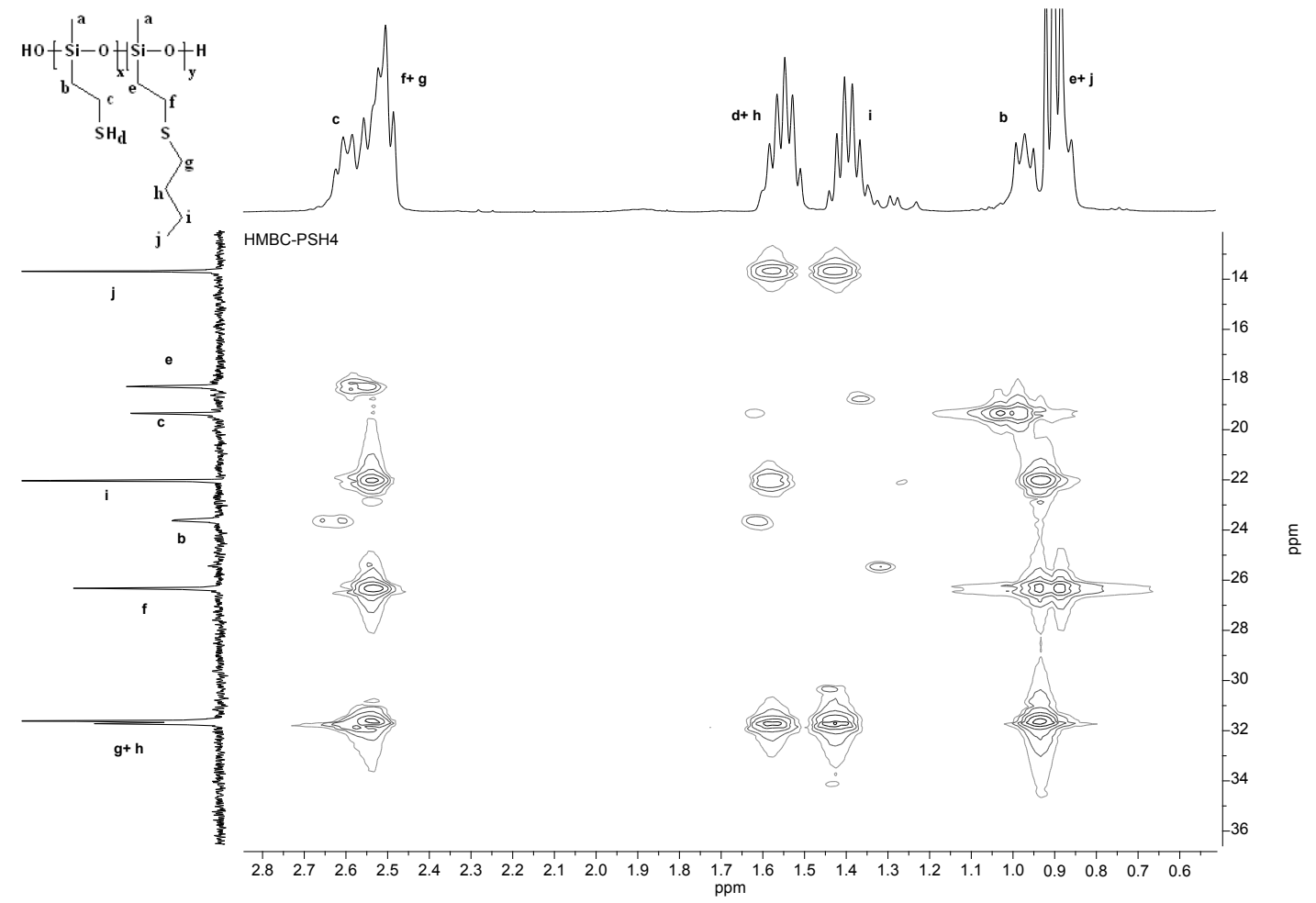

FIGURE 4 HMBC spectrum of $\mathrm{PSH}_{4}$. 
Preliminary results show aminolysis to be an alternative way to deprotect the thioestes to thiols, as it is known that butyl amine does not attack the polysiloxane chain. The signal of the methyl group from thioester in ${ }^{1} \mathrm{H}$ NMR spectrum was completely removed after $48 \mathrm{~h}$ at $65{ }^{\circ} \mathrm{C}$ (See $\mathrm{SI})$. Special care has to be however taken during this reaction to avoid formation of disulfides which leads to cross-linking. This problem might be overcome, when sacrificial 1,4-dithioerythritol is used which allows intramolecular cyclization to disulphide, which is energetically favoured since a six member ring is formed. ${ }^{22}$

\section{CONCLUSIONS}

In conclusion, the thiol-ene addition of thioacetic acid to polymethylsiloxanes containing vinyl as end- or side-groups is an efficient reaction which allows introduction of protected thiols as end- or side-groups quantitatively within few min. The amount of protected thiol side-groups into the polymers was tuned by reacting polymethylvinylsiloxane with different ratios of thioacetic acid/butanethiol, whereby butanethiol ensured a complete conversion of vinyl groups. The reduction of the thioester containing polysiloxanes by $\mathrm{LiAlH}_{4}$ allows access to polymethylsiloxanes that carry thiols either as end- or side-groups. Such polymers offer themselves for a second functionalization event whereby thiol-ene reaction can be used. Furthermore, since the butanethiol can in principle be replaced by other functional thiols, the described procedure suggests the broad applicability of the method.

\section{ACKNOWLEDGEMENTS}

We gratefully acknowledge financial support by Sciex, Swiss National Science Foundation and UEFISCDI, under the Swiss- Romanian Cooperation Program, grant No. IZERZO_142215 / 1 (10 / RO-
CH/RSRP/01.01.2013), and Swiss Federal Laboratories for Materials Science and Technology (Empa, Dübendorf). DMO and EP thank Dr. Cazacu from Petru Poni Institute for acting as home mentor of EP.

\section{REFERENCES AND NOTES}

1. M. I. Montanez, L. M. Campos, P. Antoni, Y. Hed, M. V. Walter, B. T. Krull, A. Khan, A. Hult, C. J. Hawker, M. Malkoch, Macromolecules, 2010, 43, 6004-6013.

2. A. B. Lowe, Polym. Chem. 2010, 1, 1736.

3. K. Goswami, A. L. Skov, A. E. Daugaard, Chem. Eur. J. 2014, 20, 9230-9233.

4. M. Le Neindre, R. Nicolay, Polym. Chem. 2014, 5, 4601-4611.

5. C. G. Overberger, H. Aschkenasy, J. Org. Chem. 1960, 25, 1648-1651.

6. C. G. Overberger, H. Aschkenasy, J. Am. Chem. Soc. 1960, 82, 4357-4360.

7. M. Le Neindre, B. Magny, R. Nicolay, Polym. Chem. 2013, 4, 5577-5584.

8. P. Espeel, F. Goethals, M. M. Stamenovic, L. Petton, F. E. Du Prez, Polym. Chem. 2012, 3, 1007-1015.

9. M. Le Neindre, R. Nicolay, Polym. Chem. 2014, 5, 4601-4611.

10. S. J. Dünki, Y. S. Ko, F. A. Nüesch, D. M. Opris, Adv. Funct. Mater. 2015, 25, 2467-2475.

11. S. J. Dünki, M. Tress, F. Kremer, S. Y. Ko, F. A. Nüesch, C.-D. Varganici, C. Racles, D. M. Opris, RSC Advances, 2015, 5, 50054-50062.

12. J. Mui, Q. Yick Pui, D 2300912, 1973; C. Piskoti, US 4,622,412, 1984.

13. M. A. Cole, C. N. Bowman, J. Polym. Sci. Part A: Polym. Chem. A, 2012, 50, 43254333.

14. O. van den Berg, L.-T. T. Nguyen, R. F. A. Teixeira, F. Goethals, C. Özdilek, S. Berghmans, F. E. Du Prez, Macromolecules, 2014, 47, 1292-1300. 
This document is the accepted manuscript version of the following article:

Perju, E., Dünki, S. J., \& Opris, D. M. (2016). A versatile synthetic path to thiol containing polysiloxanes. Journal of Polymer Science. Part A: Polymer Chemistry, 54(18), 2940-2948. http://doi.org/10.1002/pola.28179

15. Hoyle, C. E.; Bowman, C. N. Angew. Chem., Int. Ed. 2010, 49, 1540-1573.

16. P. V. Bonsignore, C. S. Marvel, S. Banerjee, J. Org. Chem., 1960, 24, 237240.

17. E. Klemm, U. Beil, R. Märtin, Acta Polymerica, 1991, 42, 20-22.

18. A. Berger, US 3,692,812, 1972.

19. M. A. Cole, C. N. Bowman, J. Polym. Sci. Part A: Polym. Chem. 2013, 51, 17491757.

20. M. Roth, A. Oesterreicher, F. H. Mostegel, A. Moser, G. Pinter, M. Edler, R. Piock, T. Griesser, J. Polym. Sci. Part A: Polym. Chem. 2016, 54, 418-424.

21. W. Noll, Chemistry and Technology of Silicones, Academic Press, New York and London, 1968.

22. S. Ghosh, S. Basu and S. Thayumanavan, Macromolecules, 2006, 39, 5595-5597. 
Elena Perju, Simon J. Dünki, and Dorina M. Opris

The thiol-ene addition of thioacetic acid to vinyl containing polysiloxanes allows introduction of protected thiols as end- or side-groups quantitatively within few min. The thioesters are subsequently deprotected to the respective thiols by reduction with $\mathrm{LiAlH}_{4}$. The resulting thiols can be used for another functionalization.

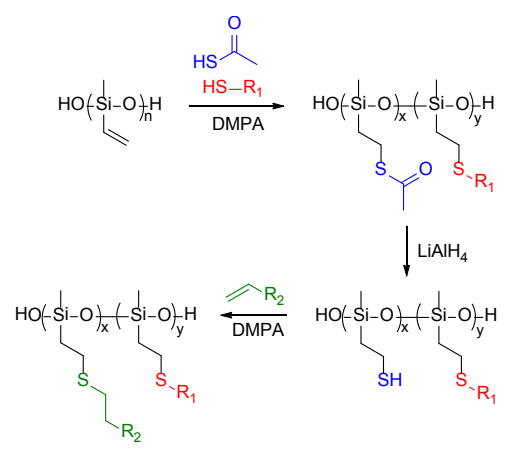

